\title{
Predicting zoonoses
}

\author{
Karl Gruber
}

\begin{abstract}
As the line dividing human and wild habitats becomes thinner, we might be brewing the world's next big pandemic. Zoonoses are diseases that are naturally transmitted between animals and humans, and a new project aims to predict their occurrence.
\end{abstract}

ast year a study identified the source of a rare retrovirus called human T-lymphotropic virus 4 (HTLV-4) that crossed the species boundaries and infected humans ${ }^{1}$. The virus had previously been reported in gorillas from Cameroon, explains Antoine Gessain, head of the Oncogenic Virus Epidemiology and Physiopathology Unit at the Pasteur Institute, who led the study. "After analysing by molecular means, blood samples from 300 people bitten by a non-human primate and living in villages and settlements in the forest of Central Africa, we were able to identify two hunters from Gabon who carried the virus." Both reported having been severely bitten by a gorilla during hunting activities in the past.

The research suggests a zoonotic origin for the virus, reinforcing current thinking that gorillas are important viral reservoirs potentially affecting humans and offering a stark reminder of the potential of such zoonotic spillovers.

About 100 years ago another virus made several independent jumps from chimpanzees and gorillas into humans in western Central Africa. The human version of this virus has killed over 35 million people over the past 30 years, and its name will be very familiar: the human immunodeficiency virus (HIV), the infectious agent behind AIDS. Today, more than 36 million people live with HIV, according to WHO estimates ${ }^{2}$.

A more recent example is the Zika virus, which in 2015 was responsible for unprecedented numbers of infections in pregnant women, causing their unborn babies to develop microcephaly ${ }^{3}$. The virus was first discovered in 1947, in the Zika forest of Uganda when it infected a caged rhesus macaque, part of a study. Some years later the first human reports of Zika virus started to appear.

But humans are not the only victims of the killing scourge caused by these viruses. In November 2016, the Netherlands killed over 190,000 ducks, Sweden 200,000 chickens, Germany about 100,000 turkeys and chickens, and more recently

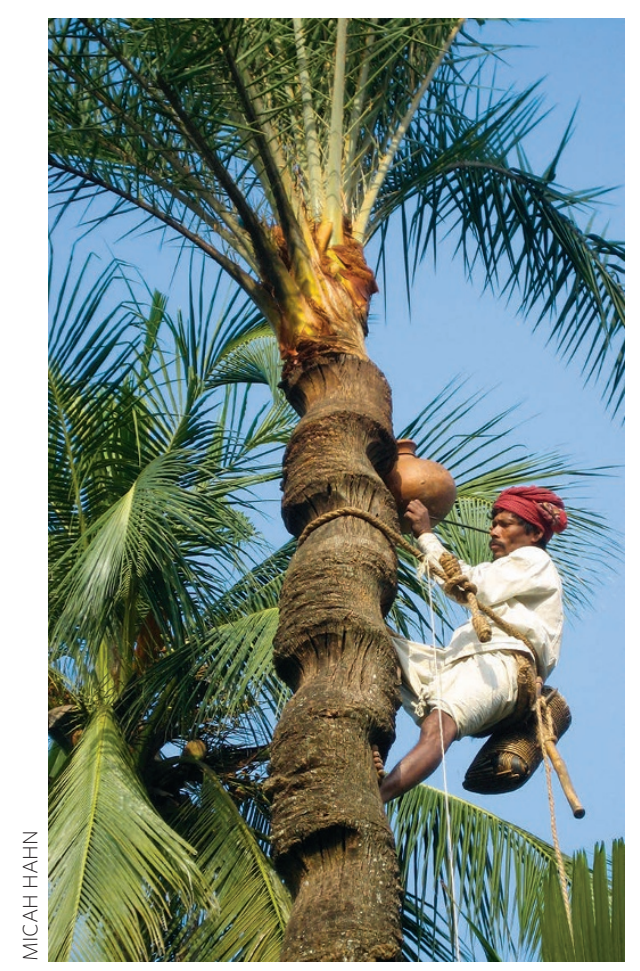

Figure 1 | A gachi, or palm sap collector, hanging a collection pot in Faridpur District, Bangladesh. Fruit bats often help themselves to the sap collected in these pots and, in the process, end up contaminating the sap with their urine, faeces, or saliva.

South Korea has killed about 7 million chicken and ducks. Why? Because of an influenza scare. The H5N8 influenza virus was detected in these unfortunate birds and culling was the best way to eliminate the virus from the population and prevent further spread, explains Thomas C. Mettenleiter, president of the Federal Research Institute for Animal Health in Greifswald-Insel Riems, Germany.

"The destruction of the captive birds from infected holdings is performed to eliminate the virus from the poultry population and prevent further spread (and even more killings)," says Mettenleiter.
Luckily for us, H5N8 is so far only known to infect birds. But other influenza strains have made the jump into humans, such as H5N1, H7N3, H7N7, H7N9, H9N2 and H1N1. The infamous H1N1 strain of 1918 infected nearly 500 million people, killing at least 50 million. A similar strain made a comeback recently, between April 2009 and August 2010, causing 18,500 laboratoryconfirmed deaths and as many as half a million deaths related to the disease around the world, according to a 2012 report ${ }^{4}$.

Poultry and primates are not the only species we should be concerned about when it comes to viral reservoirs. Viruses of all sorts, some pathogenic, some innocuous, live in myriads of different animals, from primates and rodents, to birds, bats and pigs. Bats are a particular concern: SARS, Middle East respiratory syndrome (MERS), Nipah, Marburg, Hendra, rabies, Duvenhage, Kyasanur Forest virus, Menangle, Tioman, Melaka, and lyssavirus all came from bats, and Ebola might have too, although this is not confirmed yet ${ }^{5}$.

All these animals have served as reservoirs where one or more viruses have found safety, alliance or simply managed to survive. But the odd part of the story behind many of these diseases is that the virus involved has probably been around for a very long time, living peacefully in its animal reservoir, causing no harm to beast or man. Why then, do they make the jump to the dark side? What ecological and evolutionary steps do these viruses take to become the next big pandemic?

\section{Ecology of a killer}

One of the deadliest, fastest spreading and most impactful zoonotic outbreaks of modern times started with food, in China's culinary capital. Guangdong is one of the largest and wealthiest provinces in China, home of Cantonese cuisine. However, in 2002 Guangdong was at the centre of a ghastly event: it became the birth place of the twenty-first century's first global pandemic.

Severe acute respiratory syndrome (SARS) is caused by the SARS coronavirus 




Figure 2 | Adult male mandrill hunted in Gabon to be sold for food.

(SARS-CoV) and starts as an atypical pneumonia, with high fever and mild respiratory symptoms, which quickly evolves into a full-blown pneumonia. The disease kills about $10 \%$ of all infected people and, from February 2002 to July 2003, 8,906 people acquired SARS, leading to over 774 deaths in 29 countries, according to WHO figures. The disease outbreak was officially over by July 2003 , thanks to the efficient containment efforts of the WHO and governments around the world. Sometime afterwards three independent research papers pointed to coronaviruses as the culprits behind this disease $e^{6-8}$. But the question of the ecology and evolution of the virus still remained unanswered.

Where did the virus come from and how did it get into humans? Shortly after the SARS pandemic was under control, the WHO created a special mission assigned to uncover the natural host of the virus. The international team got to work in Guangdong, where everything started.

The first clue came from a chef in Shenzhen who was one of the first persons infected. He worked at a restaurant serving exotic game food, where he was in regular contact with live caged animals. People in Guangdong, as in many places in China, appreciate their meat fresh, but not fresh as in freshly stocked in a supermarket, explains Linfa Wang, director of the Programme in Emerging Infectious Disease at Duke-NUS Medical School, Singapore. "In Guangdong you eat your meat freshly slaughtered," he says. Live animals can be bought from hundreds of small markets that keep all sorts of animals in cages, ready to please any palate.

A team led by Yi Guan from the University of Hong Kong took samples from several animals, from poultry and ferrets to snakes, turtles and feral cats. With genomic tools, the team was able to identify palm civets as the most likely source of SARS$\mathrm{CoV}$. "However, this small mammal was unlikely to be the primary reservoir of the virus as only civets in market, but not in farms, are positive for SARS-CoV", says Wang. Instead, his "gut feeling," together with some preliminary findings and his many years of experience working with the bat-borne Hendra virus in Australia, led him to bet on bats as the natural host. In 2005, Wang's international team published a study settling the question and pointing to horseshoe bats as the likely reservoir ${ }^{9}$.

"Although the bat-borne viruses were not identical to the human SARS-CoV, their close genetic relatedness and the fact that there were more than one such SARS-like viruses present in bats made us to conclude that bats are the most likely reservoir hosts of SARS-CoV at the time of the discovery," said Wang.

As for the question of how bats and civets met, the most likely scenario involved the overcrowded live animal markets, says Wang. "From farming, wholesale to retail and restaurant, there is ample opportunity for mixing of different animals including civets and bats. In the live animal market in Shenzhen, there were more than 130 different species of animal traded under the same roof in a big shed market," he says. All you needed was a few infected bats added to the mixture.

\section{To evolve or not to evolve}

SARS-CoV is a large, single-stranded RNA virus that, like all the members of its genus (Coronavirus) is known to infect both humans and animals. The name comes from their appearance under an electron microscope: a cell surrounded by projections that look a bit like a royal crown, or corona in Spanish. These crown-like structures are proteins known as viral spikes or 'S' glycoproteins and are the key factors determining if the virus can invade a new cell, interacting with specific cell receptors in a lock and key fashion.

When SARS-CoV jumped from horseshoe bats into palm civets, specific changes in these $\mathrm{S}$ proteins occurred that allowed the virus to infect the cells of the new host, first of civets and then of humans, explains Michael Farzan, a virologist and professor at the Department of Immunology and Microbial Science at the Scripps Research Institute, Florida, USA.

"What we know is that SARS coronavirus circulating in the Guongdong exotic animal marketplace was in general not particularly dangerous, but, perhaps by random mutation, or some level of selection in palm civets, it acquired two key mutations in the receptor-binding domain of its $S$ protein that allowed it to use the human orthologue of the receptor ACE2 with high efficiency." Coronarivuses are not alone in this evolutionary pattern, notes Farzan: "We've also observed a similar pattern with New World arenaviruses that cause severe haemorrhagic fevers in humans (Junin, Machupo, Guanarito)."

Viruses like SARS-CoV or MERS made their way into humans by finding an intermediate host that allowed them to multiply and prosper, and evolve to a form that was more amenable for a human host. Other viruses rely on their speedy evolutionary clock, which allows them to accumulate mutations much faster than any other organisms on Earth.

"Evolution is usually a slow process and for mammals and other animals it typically takes thousands of years before observable changes occur. But for viruses, the rate of evolution is highly accelerated and so evolutionary change can occur over much shorter time scales - even within a few days," says Fabian Leendertz, team leader of the Epidemiology of Highly Pathogenic Microorganisms group at the Robert Koch Institute in Berlin.

Such speedy evolution is what allowed viruses like HIV-1 and Zika to infect so many people, and evade not only our body's immune system but also our efforts to design an effective vaccine. The HIV-1 virus, in fact, might be the world's fastest evolving system, according to a recent report ${ }^{10}$.

But evolution is not always a necessary step, sometimes ecology is enough. Take the Nipah virus outbreak, which started in the Malaysian town of Sungai Nipah in 1999 and infected 283 people. The outbreak led to the killing of nearly 1.1 million pigs across the area of infection to prevent further 


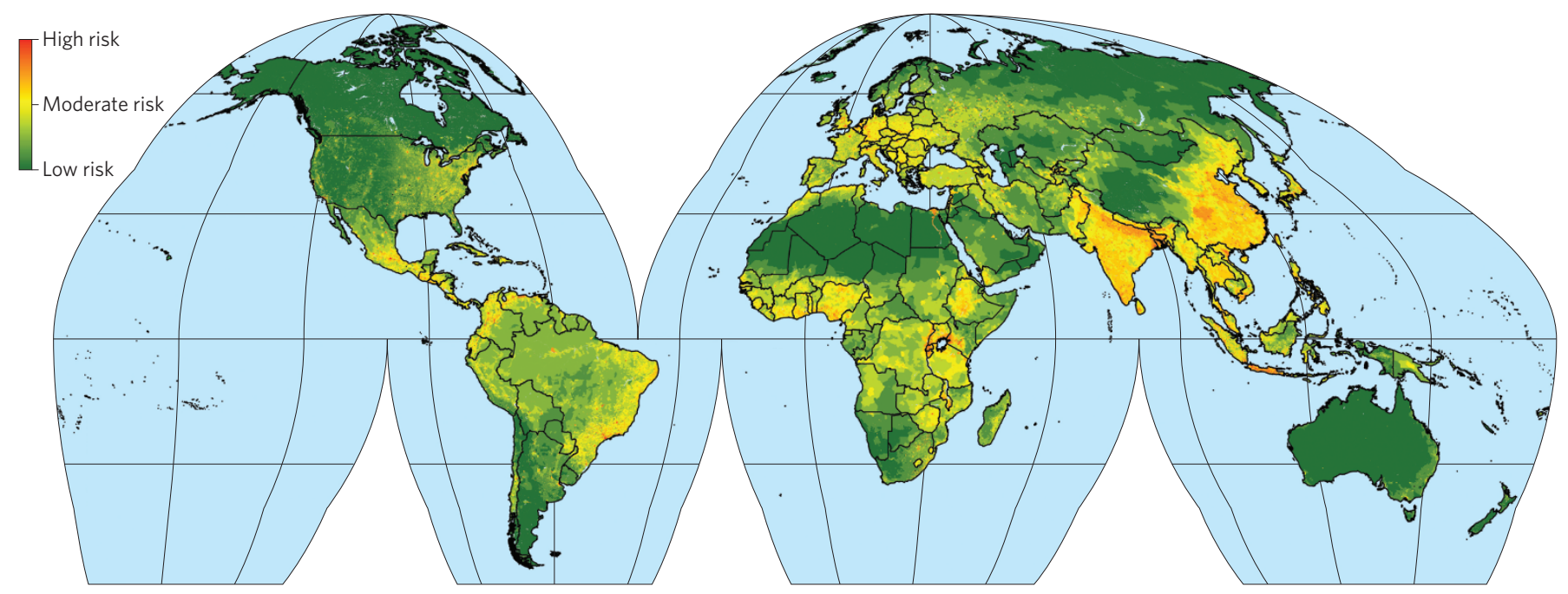

Figure 3 | Hotspot map showing predicted relative risk of zoonotic events after correcting for reporting biases. Data from ref. ${ }^{12}$, EcoHealth Alliance/PREDICT Consortium.

spread of the virus, which in this case killed 107 humans. This virus came from fruit bats and although pigs were involved in the jump to humans, they were not the only culprit.

Epidemiological studies in Bangladesh (a nearly pig-free country) traced down the bat-human route of infection to a very different source: date palm sap (Fig. 1). In the Tangail District of central Bangladesh, people drink the raw juice of date palms, normally procured by gachis or tree people (from the Bangla word gach, meaning tree). The tree is also a favourite feeding spot for fruits bats, and hence where the trouble brews. For Nipah, a sip of soiled date-palm sap is all you needed to get infected. No evolution required ${ }^{11}$.

"At least for the henipaviruses in Asia and Australia, there doesn't seem to be much 'adaptation'. The Nipah viruses from the fruit bat reservoir, pigs and humans are almost identical. Unlike the SARS story, the ephrinB2 receptor is extremely conserved and studies have shown that the $\mathrm{NiV}$ envelope ( $\mathrm{G}$ attachment protein) binds to and uses ephrinB2 equally well from multiple mammalian species," explains Benhur Lee, a virologist from the Icahn School of Medicine at Mount Sinai, New York.

"I think the spill-over cases mostly resulted from factors that allowed opportunities for humans to get in contact with large doses of viruses. Henipaviruses, despite their high mortality rates, are not easy viruses to transmit. It probably requires intimate/repeated contact with bodily fluids (saliva, urine) containing large amounts of viruses," says Lee.

Nipah is not alone in this pattern, other viruses such as HIV or HTLV didn't need to evolve either before making the jump into humans. And, while it is clear that both ecology and evolution play a role in the dynamics of a zoonotic outbreak, one important question remains: when will the next big zoonotic outbreak occur and how many people will die this time?

Using tools from the realms of ecology and evolution an innovative and massive project is seeking to answer this very question.

\section{The PREDICT project}

Pandemics of zoonotic origins should be rare, at least in theory, because they require a series of specific and unusual events, such as the interaction between two species from distinct ecological niches or the adaptation of viral strains to a new host. Yet, several zoonotic outbreaks have occurred in the past few decades alone and today many scientists are asking why and wondering if we can predict the next pandemic.

The PREDICT project, based at the One Health Institute, University of California, Davis, is the world's largest effort to track down emerging zoonotic diseases. It involves a small army of 2,500 scientists, government personnel, physicians, veterinarians, resource managers, laboratory technicians, hunters, and students. The project, spread over more than 30 countries around the world, procured tissue samples from a large variety of vertebrate animals that could serve as reservoirs of human infectious agents. PREDICT seeks to identify the viruses or other microorganisms found in wild animals with the goal of making a highly educated guess of where the next zoonotic outbreak could occur ${ }^{12}$.
The project has analysed samples from over 56,000 wild animals, including nonhuman primates, bats, rodents and other wild species used in the bush meat trade (Fig. 2). So far, over 800 novel viruses that have the potential to infect humans have been identified, half of which were found in bats, 234 in non-human primates and 143 in rodents and shrews.

In Peru, for example, PREDICT identified a broad spectrum of zoonotic pathogens, from bacteria to viruses, living in wildlife being traded in public markets. "Amazonian countries are endemic to a large number of tropical diseases, many of them naturally circulating in wildlife in the forests. Unfortunately, our surveillance of wildlife-borne zoonotic disease is still weak. In my personal opinion, if wildlife trafficking continues being an unattended problem in the region, the next pandemic may arise right here," says Ana Patricia Mendoza, from the IUCN Species Survival Commission (SSC) Wildlife Health Specialist Group, who worked with the PREDICT project.

But, can we really predict the next zoonotic pandemic with the current knowledge base and technological platforms? Many experts cast doubt with that notion. "If we can't even predict the common flu, how can we expect to predict a more complicated disease with as yet unknown pathogens?" asks Wang

At the heart of the problem is the unpredictability of these zoonotic events. "Zoonotic spill-over is largely by chance events, so there is inherent unpredictability in infections jumping across species. Much then comes down to rate of interaction with humans, which is going up for pretty 
much all potential reservoir species," says Steven Belmain, professor of ecology at the Natural Resources Institute, University of Greenwich, London.

Jonna Mazet, principal investigator at PREDICT, agrees with these sombre comments, except the part about events being by chance, she clarifies.

It is a tough job, riddled by invisible pathogens that could jump into humans at any time anywhere in the world. But, as Mazet argues, a first step is to identify areas of high risk.

"We aren't yet ready to predict the next zoonotic event, we aren't close to that yet because we don't have the data on which to base those predictions. That is why we need to do the hard work, such as is just being started by our PREDICT project. Our team is working diligently to begin to collect the data that will change this responsive, and somewhat hopeless, paradigm. Prediction will likely become more feasible as we collect and interpret the data collaboratively across the globe on the sharing of viruses between people and animals and all of the risk information surrounding these exposures that can help us forecast and mitigate spillover," she says. "As we learn how best to gather the necessary samples and analyse them, we will provide those most underserved communities the ability to detect and diagnose zoonotic threats in local laboratories to halt the spread of disease at the source of spillover and to understand exposure risks, so that local changes in behaviour can be implemented to reduce pathogen spill-over, amplification, and spread."

All in all, a take-home message when thinking about zoonotic outbreaks concerns our place within delicate ecosystem we live in, says Leendertz. "Viruses and other microorganisms live all around us, and many even within us. They are part of this world and while some of these microorganisms are potential pathogens most live in peace, and we can't simply get rid of them all. The most important thing we can do is be ready, so that whenever a new zoonotic disease emerges, we are able to quickly identify the pathogens involved and their reservoirs, understand the triggers for spill-over events and, ultimately, contain the disease. This will need to be an integral part of national surveillance systems, especially in countries with high risk of disease emergence," he says.

Undoubtedly, some parts of the world are more likely than others to have the ideal conditions to start an epidemic, but if we are ready to tackle the outbreak as soon as it starts, it could make a big difference (Fig. 3). As Mazet says, the information from PREDICT "is helping in the design of community engagement and education to empower people at highest risk to take control of their health locally, rather than being completely dependent on international response organizations when the worst scenario becomes a reality."

Karl Gruber is a freelance science writer based in Perth, Australia.

e-mail:karlgruber@gmail.com

\section{References}

1. Léa, R. et al. Clin. Infect. Dis. 63, 800-803 (2016).

2. HIV/AIDS fact sheet. WHO (2016); http://go.nature. com/2lC8nkX

3. Nitwara, W. \& Smith, D. R. Lancet Infect. Dis. 16, el19-e126 (2016)

4. Dawood, F. S. et al. Lancet Infect. Dis. 12, 687-695 (2012).

5. Quammen, D. Spillover: Animal Infections and the Next Human Pandemic (W. W. Norton \& Company, 2012)

6. Guan, Y. et al. Science 302, 276-278 (2003).

7. Rota, P. A. et al. Science 300, 1394-1399 (2003)

8. Ksiazek, T. G. et al. N. Engl. J. Med. 348, 1953-1966 (2003).

9. Li, W. et al. Science 310, 676-679 (2005).

10. Cuevas, J. M. et al. PLoS Biol. 13, e1002251 (2015).

11. Chua, K. B. et al. Science 288, 1432-1435 (2000).

12. PREDICT Consortium Reducing Pandemic Risk, Promoting Global Health (2014).

Competing interests

The author declares no competing financial interests. 